Service social

\title{
Adapter l'intervention de groupe aux besoins des adultes alcooliques ou toxicomanes, un défi
}

\section{Natalie Bibeau}

Volume 46, numéro 2-3, 1997

Groupes - Symposium 1997

URI : https://id.erudit.org/iderudit/706770ar

DOI : https://doi.org/10.7202/706770ar

Aller au sommaire du numéro

Éditeur(s)

École de service social de l'Université Laval

ISSN

1708-1734 (numérique)

Découvrir la revue

Citer cet article

Bibeau, N. (1997). Adapter l'intervention de groupe aux besoins des adultes

alcooliques ou toxicomanes, un défi. Service social, 46(2-3), 261-273.

https://doi.org/10.7202/706770ar d'utilisation que vous pouvez consulter en ligne.

https://apropos.erudit.org/fr/usagers/politique-dutilisation/ 


\section{Adapter l'intervention de groupe aux besoins des adultes alcooliques ou toxicomanes, un défi}

Natalie BIBEAU

Ce texte se présente comme une réflexion sur l'intervention auprès des groupes ainsi que sur le service social des groupes. II s'agit en fait d'une analyse critique fondée sur une expérience clinique de plusieurs années d'intervention de groupe effectuée auprès d'adultes hommes et femmes souffrant de problèmes d'alcoolisme ou de toxicomanie. Ces séances de groupe se sont déroulées dans une clinique interne multidisciplinaire d'un centre public de réadaptation spécialisé dans le traitement de cette problématique. L'intervention de groupe est une approche privilégiée par le centre. On a constaté que plusieurs interventions de groupe sont apparues positives et efficaces, alors que d'autres se sont révélées plutôt mal adaptées aux besoins et aux champs d'intérêt des membres. C'est donc à partir d'une réflexion critique de certaines pratiques actuelles (choix de l'approche théorique, conditions organisationnelles, etc.) que des pistes de travail seront suggérées. Celles-ci visent à améliorer l'efficacité du travail de groupe ainsi que la participation des membres.

\section{CONTEXTE DE L'INTERVENTION ET CARACTÉRISTIQUES DE LA CLIENTÈLE}

Pour amorcer la réflexion, il est important de préciser dans quel contexte l'intervention de groupe étudiée s'est effectuée ainsi que certaines caractéristiques de la clientèle concernée. En premier lieu, 
les adultes présentant des problèmes d'alcoolisme ou de toxicomanie qui consultent la clinique interne de ce centre public le font de manière volontaire. Les services sont gratuits et les programmes de réadaptation sont offerts par des professionnels de diverses formations dans un cadre de pratique multidisciplinaire. Le problème de l'alcoolisme et de la toxicomanie est abordé sous un angle biopsychosocial. Selon les données du groupe Recherche et intervention sur les substances psychoactives - Québec (RISQ) (1993), la clientèle du centre est composée de $70 \%$ d'hommes et de $30 \%$ de femmes et l'âge moyen est de 33,9 ans. Les personnes qui s'adressent au centre sont en majorité polytoxicomanes. L'alcool reste le produit le plus souvent désigné comme problématique (seul ou associé à d'autres substances), suivi de la cocaiine. Le niveau moyen de scolarité est de 10,7 années. On note que $95 \%$ des personnes formant la clientèle déclarent avoir eu, au cours de leur vie, une période importante (ne relevant pas de la consommation de drogue ou d'alcool) durant laquelle elles ont souffert de problèmes psychologiques (dépression grave, anxiété grave, hallucinations, comportements violents, prise de médicaments pour problèmes émotionnels, idéations suicidaires, etc.). Plus de deux personnes sur cinq ont déjà fait au moins une tentative de suicide dans leur vie (55\% chez les femmes et $33 \%$ chez les hommes). On observe que $70 \%$ de la clientèle déclare avoir déjà été arrêtée et inculpée pour un délit criminel. Mentionnons que $40 \%$ des personnes qui demandent de l'aide ont connu des conflits graves avec leur famille durant le mois précédant l'admission. Enfin, soulignons qu'il a fallu en moyenne 13 années de consommation régulière d'alcool (c'est-à-dire au moins trois fois par semaine jusqu'à intoxication) avant que les clients et les clientes fassent une demande d'aide au centre. Ce délai est de 4,6 années pour la cocaiine.

De façon plus générale, la population alcoolique et toxicomane est considérée comme une population vulnérable. Gitterman et Shulman (1994) parlent de cette population en spécifiant qu'elle est dans des conditions de vie difficiles et opprimantes, sur lesquelles elle a souvent peu de contrôle. Les problèmes de ces personnes peuvent apparaître souvent impossibles à traiter, parce qu'ils sont chroniques, aigus et persistants. Avec le manque de soutien de la famille et de la communauté, ces personnes risquent une détérioration physique, psychologique et sociale et deviennent extrêmement 
vulnérables à l'isolement social et émotionnel. Le sentiment d'échec et la rationalisation sont également des caractéristiques importantes de cette population (Zastrow, 1993).

\section{PeRtinenCe de L'INTERVENTION DE GROUPE POUR LES POPULATIONS ALCOOLIQUES OU TOXICOMANES}

Plusieurs praticiens en alcoolisme et toxicomanie désignent le groupe comme étant la modalité de choix pour le traitement des personnes présentant des problèmes d'abus de substances (Levine et Gallogly, 1985; Cooper, 1987; Rogers et McMillin, 1989; Cavaiola, 1984 dans Milgram et Rubin, 1992). En effet, le travail de groupe est jugé très efficace pour surmonter la résistance, offrir l'occasion de mettre en place un réseau de soutien et un lieu servant à l'acquisition de comportements sociaux et interpersonnels plus appropriés (Brady, 1989). Les individus aux prises avec des problèmes d'alcoolisme ou de toxicomanie sont souvent socialement isolés de contacts sains et fonctionnels (Milgram et Rubin, 1992). Le groupe peut devenir alors une forme unique et puissante d'interactions sociales à travers lesquelles une personne alcoolique ou toxicomane peut apprendre comment les autres perçoivent ses attitudes, ses idées et ses émotions (Elder, 1990). Le groupe peut servir également à normaliser la situation de consommation, à déculpabiliser, à briser l'isolement, à partager des expériences vécues et à augmenter le sentiment de compétence.

Diverses approches d'intervention de groupe ont été conçues ou adaptées pour cette population. L'approche médicale (inspirée du milieu psychiatrique classique), le modèle des douze étapes, l'approche systémique, les techniques de confrontation, l'éducation structurée en sont quelques exemples. Chacun de ces modèles possède des forces et des limites. Il importe donc, pour l'intervenant en travail social, de trouver un modèle qui s'adapte bien au groupe visé et qui convient à son style personnel d'action.

\section{ANALYSE DE L'APPROCHE THÉORIQUE DOMINANTE}

Le programme d'intervention de groupe que nous analysons a été mis en œuvre auprès de plusieurs groupes venus en traitement à la clinique interne du centre de réadaptation. Chacun de ces groupes comptait en moyenne 13 participants. Les groupes étaient mixtes et 
fermés, c'est-à-dire qu'aucun nouveau membre ne s'ajoutait à la séquence des activités. Le programme pouvait totaliser jusqu'à 17 rencontres.

L'approche théorique dominante concernant ce programme de groupe s'apparentait davantage au modèle de l'éducation structurée. À l'intérieur de ce type de modèle, l'accent est mis sur l'apprentissage et non sur le traitement. Le travailleur social prépare un plan et un programme pour le groupe, et les membres n'ont pas à déterminer ou à élaborer ce programme (Papell et Rothman, 1983). L'ensemble des activités a donc été conçu par un groupe d'intervenants, sans l'aide ou la consultation de la clientèle alcoolique ou toxicomane. Plusieurs activités de groupe visaient à aider les participants à examiner les problèmes de leur vie à partir de thèmes. Le contenu de ces activités demeurait essentiellement informatif et construit principalement sur une base théorique choisie. Ainsi, par exemple, l'activité sur le thème de la " motivation » reposait principalement sur l'apprentissage du « modèle de changement » élaboré par Carlo C. DiClemente et collab. (1991). Ce modèle comprend cinq stades de changement (" précontemplation », « contemplation », « préparation », " action » et « maintien ») qui avaient été adaptés, par les intervenants du centre, à la problématique de l'abus d'alcool et de drogues. De la même façon, la séance de groupe portant sur le thème de la « dépendance " se basait principalement sur l'explication du cycle de l'assuétude de Stanton Peele (1982). Pareillement, les diverses activités en lien avec le thème de la " santé » visaient principalement à offrir de l'information en ce qui a trait à l'alimentation, au sommeil ou encore aux maladies transmises sexuellement. Par ailleurs, certaines autres activités s'inspiraient davantage de l'expérience personnelle des participants. En fait, le contenu des groupes d'éducation structurée peut non seulement être informatif, mais posséder aussi un volet affectif (Apgar et Coplon, 1985). Ainsi, en plus d'être didactiques, les méthodes utilisées peuvent se baser sur l'expérience vécue. De fait, certaines des activités de groupe offertes sur l'unité comprenaient des exercices de «partage du vécu ». Les échanges qui s'effectuaient dans ces activités se faisaient en petites équipes, de façon très structurée, à partir de questions bien précises. Par exemple, au cours d'une activité portant sur l'anxiété, les participants et participantes devaient, en équipe de trois ou quatre 
personnes, discuter des manifestations physiques et psychologiques de l'anxiété dans leur vie.

Mis à part ces discussions en petits groupes, très peu d'échanges avaient lieu entre les participants durant les activités. La communication se faisait donc de l'intervenant à un membre ou à l'ensemble du groupe. Les participants s'adressaient habituellement à l'intervenant, qui occupait par conséquent un rôle central. Les insatisfactions, les désaccords ou les conflits se traitaient à l'extérieur des activités de groupe, avec un intervenant et les personnes concernées. En fait, les groupes d'éducation structurée ne sont pas basés sur l'utilisation de techniques qui explorent les conflits, encouragent "l'insight » ou interprètent les comportements négatifs (Apgar et Coplon, 1985).

En dépit de cela, le modèle d'éducation structurée a répondu, sans aucun doute, à certains besoins qu'éprouvait la clientèle, dont : recevoir de l'information, comprendre davantage son problème d'alcoolisme ou de toxicomanie, être en contact avec d'autres personnes qui éprouvaient les mêmes difficultés et développer la motivation nécessaire pour arrêter la consommation. Toutefois, ce modèle présente certaines limites qui ont amoindri les possibilités qu'offre l'intervention de groupe auprès de cette population. D'abord, chacune des séances de groupe comprenait des objectifs spécifiques qui étaient imposés aux membres. Les participants ne pouvaient donc pas, comme le propose Heap (1987), rejeter ou modifier ces objectifs. Ainsi, l'absence de discussion autour des objectifs peut donner l'impression à la clientèle qu'elle n'est pas, de façon précise, concernée par le contenu des activités. Les objectifs imposés ne font pas nécessairement l'unanimité et les objectifs personnels doivent être fondus dans l'objectif collectif prédéterminé (Papell et Rothman, 1983). Dans ce type d'approche, le plein potentiel des membres n'est pas vraiment exploité, le partenariat travailleur social/membre n'est pas favorisé, les contacts et le développement de l'aide mutuelle ne sont pas encouragés. De plus, la formule d'éducation structurée peut parfois s'apparenter à de l'enseignement scolaire et devient une source d'anxiété majeure pour les membres qui ont vécu de nombreux échecs sur ce plan. Le choix d'un modèle d'intervention devient donc un élément important qui peut influer sur les possibilités de croissance offertes par le travail de groupe. 


\section{LES DIVERS OBSTACLES}

En plus du choix difficile du modèle d'intervention, d'autres obstacles peuvent rendre le travail d'intervention auprès des groupes ardu. La préparation des groupes, leur composition et le niveau de " perméabilité " des individus au traitement en sont des exemples.

II arrive que l'intervention de groupe soit perçue comme un moyen économique de desservir plus de personnes. Cette position est souvent influencée par des préoccupations administratives visant à démontrer l'efficacité et la rentabilité des services rendus à une population (Home, 1994). Ce type de préoccupation néglige parfois l'importance de la préparation des groupes. On semble oublier que les groupes mis sur pied sans préparation appropriée risquent d'échouer (Douglas, 1979). En fait, entreprendre un programme d'intervention de groupe sans préparation place les membres du groupe, de même que les intervenants, dans des conditions hasardeuses ne permettant pas toujours aux intéressés de profiter pleinement de cette expérience de groupe.

Certains aspects ne doivent pas être négligés dans la préparation et l'élaboration d'un groupe. Le recrutement et les rencontres prégroupe sont ainsi des éléments essentiels à une bonne préparation du travail de groupe. Ces rencontres prégroupe permettent de préciser l'intérêt de la personne à participer, les objectifs personnels qu'elle souhaite atteindre ainsi que les besoins particuliers qu'elle éprouve. Cette étape aide également le travailleur social ou la travailleuse sociale à évaluer la pertinence et la possibilité pour chaque individu de contribuer à l'expérience et de retirer quelque chose de ce groupe. Wickham (1993) précise que les entrevues prégroupe servent à évaluer, à informer, à calmer les peurs et l'anxiété. Elles contribuent également à amorcer l'établissement d'un climat de confiance ainsi que le développement d'une compréhension du participant éventuel.

Par ailleurs, certains organismes ou certains intervenants ne s'investissent pas du tout dans la préparation d'un groupe. II semble exister une certaine confiance à l'égard des « pouvoirs curatifs magiques qui entrent en scène par le seul fait d'être en groupe ", peu importe que l'on soit préparé ou non (Heap, 1987). On laisse ainsi au hasard le choix de la composition du groupe. C'est principalement de cette façon que les groupes étaient formés à l'intérieur du 
programme étudié. En fait, les participants étaient regroupés au hasard selon leur date de demande d'aide et les places disponibles dans les services. Aucune entrevue prégroupe n'était effectuée. Les clients étaient simplement avisés qu'ils devaient participer à des activités de groupe durant leur séjour dans les services internes. Comme nous le savons, le hasard fait parfois bien les choses, mais le hasard peut également entraîner certains problèmes, dont la constitution de groupes très hétérogènes. Ainsi, une femme dépressive de 68 ans, un jeune adulte de 22 ans présentant des problèmes de comportement importants, un homme violent en attente de procès pour " voies de fait " sur sa conjointe, une jeune femme victime de violence conjugale venant de quitter son foyer, une personne atteinte du SIDA et une femme enceinte pouvaient tous être appelés à participer à un même groupe. La seule base du problème de toxicomanie ne permet pas, dans de telles circonstances, de rentabiliser l'intervention de groupe, les problématiques individuelles consécutives à la toxicomanie étant trop diversifiées. Ces circonstances peuvent même provoquer, pour certains membres du groupe, une répulsion à ce type d'intervention. De la même façon, les groupes pouvaient être composés de douze hommes et d'une femme. Comme le précise Home (1986a), le groupe doit être assez hétérogène pour assurer une certaine vitalité, mais il demeure important qu'il soit équilibré et suffisamment homogène pour assurer sa stabilité. La composition du groupe peut devenir un élément décisif dans le processus de développement du groupe (Heap, 1987).

Enfin, un niveau varié de " perméabilité » au traitement peut, lui aussi, devenir un obstacle au développement du groupe. La perméabilité découle de l'intérêt pour les membres du groupe à s'engager, du sens de l'urgence et des besoins qui entraînent la demande (Papell et Rothman, 1990). Lorsque ces éléments se révèlent trop divergents, il devient plus difficile d'élaborer un programme et de fournir une intervention adaptée. Bien que les individus, dans la situation étudiée, aient choisi librement de participer au traitement de l'unité, certains membres présentaient des caractéristiques qui rejoignent les dimensions du travail de groupe en contexte d'autorité (voir Papell et Rothman, 1990). En effet, certaines personnes, bien que volontaires, ont pris la décision de venir en traitement parce qu'elles feraient face à de sérieuses conséquences si elles agissaient autrement (placement d'enfants, incarcération, problèmes de 
probation, etc.). L'obligation de participer est donc devenue une forme subtile de contrainte. L'attitude de ces personnes à l'égard des services est bien différente de celle de quelqu'un qui éprouve des problèmes de santé physique ou mentale importants ou qui n'en peut tout simplement plus de consommer. La motivation à consulter, le sens de l'urgence, les besoins et les intérêts qui entraînent la demande pouvaient donc varier énormément d'un individu à l'autre. Ces différences pouvaient s'avérer très positives dans certains cas et amener les gens en contexte d'autorité à réfléchir à la suite des témoignages et des contacts avec des personnes plus "volontaires ". Dans d'autres cas, la résistance pouvait devenir tellement forte qu'elle créait un climat négatif et freinait inévitablement la cohésion du groupe.

Par ailleurs, certaines personnes, bien que volontaires pour le traitement dans les services internes, n'étaient pas nécessairement intéressées au travail de groupe et préféraient une approche plus individuelle. Le fait d'obliger tout le monde à participer aux activités peut, à certaines occasions, ne pas être bénéfique. La possibilité pour un individu de pouvoir choisir librement de participer à un groupe ou non n'est pas indispensable à la réussite de l'intervention de groupe. Toutefois, une personne qui, dès le début, a le choix de participer acceptera plus facilement l'intervention de groupe. Un grand nombre de membres volontaires formeront plus rapidement un groupe efficace (Bertcher et Maple, 1974).

La préparation du groupe, sa composition, les intérêts et les besoins des membres et le choix de participer ou non au programme représentent donc quelques-uns des obstacles qui peuvent rendre l'intervention de groupe moins efficace et moins intéressante pour les membres qui y participent.

\section{VERS UNE APPROCHE ALTERNATIVE}

La population alcoolique-toxicomane peut, sans contredit, bénéficier grandement d'une intervention de groupe. Un modèle qui s'inspire davantage du potentiel de ces membres, qui encourage les contacts et le développement de l'aide mutuelle et qui met à contribution la force et la pleine capacité de l'énergie du groupe s'avérerait certainement une approche alternative intéressante ayant une portée beaucoup plus grande auprès de cette population. En fait, 
les personnes éprouvant des problèmes d'alcoolisme ou de toxicomanie venues en traitement à l'unité interne du centre de réadaptation ont démontré un grand besoin de parler de leurs préoccupations, un désir d'être écoutées et entendues. Elles ont également exprimé le souhait de mettre en place un nouveau réseau de soutien social, le leur étant parfois devenu très détérioré et limité à la suite de la consommation.

Le modèle du courant central, dans une perspective de réadaptation, du service social des groupes peut devenir une solution intéressante pour ce type de population. Le groupe devient alors un outil et offre un contexte de changement pour l'individu, tout en visant à améliorer son fonctionnement social (Vinter, 1974). L'accent est mis sur le changement individuel (Garvin et Glasser, 1974). À l'aide de ce genre de modèle, on peut viser à développer certains types d'habiletés, telles que : apprendre à communiquer, être en relation avec les autres, exprimer ses émotions, donner son opinion, signaler son désaccord, augmenter sa capacité de tolérance et d'acceptation des autres ainsi que sa capacité à se différencier (Klein, 1972 dans Wickham, 1993; Lang, 1979). Précisons que des démarches vers des relations plus tolérantes peuvent s'avérer particulièrement appropriées pour des personnes présentant des problèmes d'alcoolisme ou de toxicomanie, puisque ces dernières sont régulièrement engagées dans des relations teintées de rejet et d'intolérance. En fait, ce type de modèle peut convenir à des individus plus au moins autonomes qui ont besoin de l'aide d'un travailleur social ou d'un groupe pour résoudre certains problèmes de comportement et d'attitude (Home, 1986b). Les niveaux de structure et de confrontation peuvent varier et doivent s'ajuster à la composition, aux résistances, aux besoins et aux objectifs des membres.

Dans ce type de modèle, le travailleur social occupe une position centrale. Toutefois, même si son rôle est directif (Home, 1986b), il n'occupe pas un rôle de pouvoir. II agit comme agent de changement. En fait, le travailleur social doit voir à la structure et à la formation d'un système de communication ouvert accordant la possibilité pour les membres de contribuer au groupe, d'être entendus, accueillis et appréciés (Lang, 1979). De plus, le travailleur social encouragera la manifestation et le développement de l'aide mutuelle. 
Le principe du développement de l'aide mutuelle est certainement l'un des aspects importants du service social des groupes. En fait, le groupe a le potentiel de servir de système d'aide mutuelle pour ses membres (Shulman, 1984). Le processus d'aide mutuelle peut occasionner des expériences positives intéressantes, dont : un climat de soutien entre les membres, une cohésion, le développement de l'espoir, l'altruisme et l'existence d'un milieu où les membres peuvent tester et comparer leurs émotions et leurs perceptions (Northen, 1988 dans Fatout, 1992). Toutefois, mettre simplement des gens ensemble ne garantit pas l'émergence de l'aide mutuelle. De fait, certains obstacles peuvent gêner cette émergence. Les motivations divergentes des membres du groupe à s'engager dans le processus d'aide, l'impression pour certaines personnes d'avoir des intérêts, des besoins et des sentiments uniques et la difficulté de communiquer honnêtement en sont des exemples (Shulman, 1984). Le stress, la résistance, des modèles de communication et de relations dysfonctionnels, le déni et la non-expression de la colère sont d'autres obstacles qui peuvent nuire au développement de l'aide mutuelle (Gitterman, 1989).

Manifestement, les risques sont grands que plusieurs de ces obstacles surgissent dans des groupes formés d'individus présentant des problèmes d'alcoolisme ou de toxicomanie. Par ailleurs, considérant que les réseaux de soutien social des individus qui viennent en traitement sont souvent détériorés, le développement de l'aide mutuelle est certainement une façon stratégique et efficace d'aider les gens à s'aider. Le travailleur social pourrait donc essayer d'encourager et d'exploiter le développement d'un certain type d'aide mutuelle. Dans le travail auprès des populations alcooliques ou toxicomanes, il est impératif que le système d'aide mutuelle demeure en lien avec les normes, les valeurs et les objectifs fixés par le groupe. Ainsi, le développement d'un type d'aide mutuelle centré sur la réduction ou l'élimination de la consommation (trouver ensemble des comportements alternatifs à la consommation) et sur la modification de certains comportements spécifiques (s'aider à respecter les règles du groupe, exprimer ses émotions aux autres, prendre davantage de responsabilités, être plus intègre à l'égard des autres, etc.) pourrait être encouragé par le travailleur social et devenir un atout précieux pour l'intervention de groupe. Par ailleurs, il faudrait veiller à ce que le développement des liens et de l'entraide entre certains 
membres du groupe ne serve pas à organiser et à augmenter la résistance au traitement ni à encourager le maintien du style de vie associé à l'abus des substances. II devient donc important pour le travailleur social d'encourager, dès le début, un climat de respect qui demeure en lien avec les normes, les valeurs et les objectifs fixés par le groupe. Par ailleurs, dans ce modèle axé sur la réadaptation, bien que l'aide mutuelle puisse être encouragée et développée, elle ne devient pas la finalité du groupe (Alexandre, 1989). En fait, l'aide mutuelle est exploitée différemment selon le modèle d'intervention choisi et la dynamique du groupe en cours.

\section{CONCLUSION}

Dans une perspective de réadaptation, l'utilisation du modèle du courant central du service social des groupes peut devenir une option intéressante à employer auprès d'une clientèle alcoolique ou toxicomane. Cependant, il est parfois difficile d'appliquer de façon absolue un modèle d'intervention spécifique. La combinaison de différentes approches peut alors constituer une solution alternative sensée. En fait, la possibilité d'utiliser un modèle dominant et d'y greffer des attributs d'autres approches peut s'avérer un choix judicieux. Ainsi, certains aspects moins menaçants de l'éducation structurée pourraient être maintenus et ajoutés à un modèle principal choisi. Enfin, considérant différents facteurs, tels que l'homogénéité, l'autonomie et l'ouverture au traitement des participants, le travailleur social pourrait décider de se diriger vers une approche plus souple et davantage centrée sur la participation active des membres. Choisir un modèle d'intervention bien adapté à la population visée dans le respect de ses valeurs et de ses préférences professionnelles, préparer les membres du groupe à leur expérience et contrôler davantage la composition des groupes peuvent devenir des éléments importants dans l'atteinte de nos objectifs. L'intervention de groupe demeure un outil de travail puissant et remarquable. À nous d'en maximiser l'efficacité.

Natalie BIBEAU

Travailleuse sociale Centre hospitalier des Vallées de l'Outaouais

Chargée de cours 


\section{Références bibliographiques}

ALEXANDRE, H. (1989). L'intervention de groupe auprès des pères incestueux, Essai de maîtrise, Québec, Université Laval.

APGAR, K. et J.K. COPLON (1985). « New Perspectives on Structured Life Education Groups », Social Work, mars-avril, p. 138-143.

BERTCHER, H.J. et F. MAPLE (1974). " Elements and Issues in Group Composition ", dans P. Glasser, R. Sarri et R. Vinter (dir.), Individual Change Through Small Groups, New York, Free Press, p. 186-208.

BRADY, D.E. (1989). "Substance Abuse : The Use of Groups in Relapse Prevention ", dans Actes du Symposium : Le service des groupes et défi du changement sociétal, vol. 1, Montréal.

COOPER, D. (1987). " The role of group psychotherapy in the treatment of substance abusers ", American Journal of Psychotherapy, XLI, p. 55-67.

DiCLEMENTE, C.C. et collab. (1991). " The process of smoking cessation : analysis of precontemplation, contemplation and preparation stages of change ", Journal of Consulting and Clinical Psychology, 59, p. 295-304.

Douglas, T. (1979). Groupwork Practice, Londres, Tavistock Press.

ELDER, I.R. (1990). Conducting Group Therapy with Addicts, Bradenton, TAB.

FATOUT, M.F. (1992). Models for Change in Social Group Work, New York, Aldine de Gruyter.

GARVIN, C.D. et P.H. GLASSER (1974). " Social Group Work : The Preventive and Rehabilitative Approch ", dans P. Glasser, R. Sarri et R. Vinter (dir.), Individual Change Through Small Groups, New York, Free Press, p. 34-49.

GITTERMAN, A. (1989). «Building Mutual Support in Groups », Social Work with Groups, vol. 12, n² 2, p. 5-21.

GITTERMAN, A. et L. SHULMAN (dir.) (1994). Mutual Aid Groups, Vulnerable Populations, and Life Cycle, $2^{\ominus}$ éd., New York, Columbia University Press.

HEAP, K. (1987) (1985). La pratique du travail social avec les groupes, Paris, ESF.

HOME, A. (1994). « Réussir l'intervention de groupe malgré un contexte difficile : Mission impossible? ", dans Actes du $2^{e}$ Colloque : Les Journées Simone Paré, Daniel Turcotte (dir.), Québec, Université Laval, Cahiers du service social des groupes, IX, p. 27-40. 
HOME, A. (1986a). La formation des groupes, Montréal, Université de Montréal, doc. miméo.

HOME, A. (1986b). Tableau comparatif de 3 modèles du service social des groupes, Montréal, Université de Montréal, doc. miméo.

LANG, N.C. (1979). Some Defining Characteristics of the Social Work Group : Unique Social Form, Paper prepared for the Symposium on Social Work Practice With Groups, Cleveland.

LEVINE, B. et V. GALLOGLY (1985). Group Therapy with Alcoholics, Beverly Hills, Sage.

MILGRAM, D. et J.S. RUBIN (1992). "Resisting Resistance : Involuntary Substance Abuse Group Therapy ", Social Work with Groups, vol. 15, no 1, p. 95-110.

PAPELL, C.P. et B. ROTHMAN (1990). "Service social des groupes en contexte d'autorité ", Service social, vol. 39, n 1, p. 45-63.

PAPELL, C.P. et B. ROTHMAN (1983). " Le modèle du courant central du service social des groupes en parallèle avec la psychothérapie et l'approche de groupe structuré ", Service social, vol. 32, nos 1 et 2, p. 11-31.

Peele, S. (1982). L'expérience de l'assuétude, Université de Montréal, Faculté d'éducation permanente.

RISQ (Recherche et intervention sur les substances psychoactives Québec) (1993). Analyse descriptive de la population en traitement à partir de l'IGT 1991-1992, par Louise Guyon et Michel Landry, Montréal, RISQ.

ROGERS, R.L. et C.S. MCMILLIN (1989). The Healing Bond, Treating Addictions in Groups, New York, W.W. Norton \& Company.

SHULMAN, L. (1984) (1979). The Skills of Helping Individuals and Groups, Itasca, F.E. Peacock Inc. 